\title{
Orientation of Solfeggio Course Development in Normal Universities
}

\author{
Chao Song ${ }^{1, *}$ \\ ${ }^{1}$ School of Music, Shaanxi Normal University, Xi'an, 710119, China \\ *Corresponding author. Email: songchao@snnu.edu.cn
}

\begin{abstract}
Solfeggio, as an essential professional basic course in music teacher education system, has always been carrying the function of solving music auditory cognition and assisting all kinds of music practice. At the present stage, the definition and category, purpose and function, concept and evaluation of the solfeggio course have attracted much attention in the collaborative development of other courses. Based on the characteristics of music education in normal universities and the new pattern of teacher education system, this work re-examined the problem of solfeggio course development through the three orientations of "closely adhering to the nature of the discipline, focusing on aesthetic psychology and concentrating on auditory thinking", expecting to explore new ideas of promoting the development of solfeggio course in normal universities.
\end{abstract}

Keywords: Solfeggio in normal universities; Course development; Orientation exploration

\section{INTRODUCTION}

Solfeggio, as an essential professional basic course in music teacher education system, has always been carrying the function of solving music auditory cognition and assisting all kinds of music practice. At the present stage, with the continuous innovation of the teaching concept in the field of basic music education, the teaching task of solfeggio is also changing from bottom to top. Therefore, the definition and category, purpose and function, concept and evaluation of solfeggio course have attracted much attention in the collaborative development of other courses. This work tried to re-examine the problem of solfeggio course development through the three orientations of "closely adhering to the nature of the discipline, focusing on aesthetic psychology and concentrating on auditory thinking", expecting to explore new ideas of promoting the development of solfeggio course in normal universities.

\section{THE ORIENTATION OF CLOSELY ADHERING TO THE NATURE OF THE DISCIPLINE}

\subsection{Clarify course definition}

The essence of a discipline is the fundamental attribute of a discipline, which is mainly reflected in the following aspects: first, the research object and the basic problems of the discipline; second, the core discipline concept and category; third, the basic discipline method and thought, and its core is the discipline thinking; fourth, the core discipline value and spirit[1]. Fundamentally speaking, solfeggio discipline is committed to the development of music hearing, and this point is always beyond doubt. The core of the research objects and basic problems of the solfeggio discipline has always centered around the development of music and hearing. The discipline concept and category are also developed in some problems related to auditory psychology, and the basic discipline method and discipline value should also be closely related to the essence of discipline.

The development of solfeggio course should first be based on the development of music and hearing. Like other cognitive activities, the occurrence and development of music hearing needs to be carried by psychology, which is embodied in a series of processes, including perception, memory, imagine and thinking. The purpose of music auditory training is to make the auditory psychology complete the high summary of the auditory cognitive process under the repeated stimulation of the external audio, then form the inner hearing, and help the auditory subject obtain the ability to use the auditory appearance at will.

\subsection{Clarify category center}

In the process of auditory cognition objectification, the acquisition of musical auditory ability first comes from the decomposition of musical elements, which constitutes the main content of solfeggio teaching, namely the specific training items of pitch, rhythm, melody, interval, and chord. Mr. Bennett Reimer pointed out in his monograph The Philosophy of Music Education that music education is to carry out human sensory education through the cultivation of internal expressive response to the sound. The most important role of music education is to help students 
gradually increase their sensitivity to music elements, including melody, harmony, rhythm, timbre, weaving, and curvature, and they are all objective, which can be identified, named, used, created, discussed, extracted and inserted. The auditory aesthetic of music education is first the perception of musical elements [2].

Pitch, rhythm, speed, strength, tone, and tonality are the main elements of music, among which, the role of pitch is self-evident. The connection of transverse pitches is melody, the combination of longitudinal pitch is range and chord, and the pitch in vertical and horizontal relationship is harmony, polyphonic and texture. Jepprov has done a very deep study of music hearing problems, arguing that the broad sense of music hearing refers to the pitch, timbre and dynamic hearing, and the music hearing in the narrow sense refers to the pitch hearing [3]. This view fully affirms the value of pitch hearing in music hearing and clarifies the primary task of solfeggio teaching.

The decomposition of musical elements is mostly dominated by pitch. It is known that solfeggio training course, as a special hearing training course, needs to correspond to the pitch of the roll. Both the fixed-doh and movable-doh need to add the "shells" in the corresponding tonality to distinguish the different heights. As a music symbol system and information carrier system, solmization is an indispensable tool for music communication and expression. As a material shell with the abstract function of pitch, solmization is the main support of music thinking and has the essence and function as music "language" [4]. The decomposition of music elements is mostly reflected in the embodiment of solmization. The correspondence of roll and pitch plays a central role in the process of "listening, singing, reading and writing" in solfeggio practice, which is also the basis for the operation of musical thinking.

The development of solfeggio course should first focus on the essence of the subject, take the construction of music auditory psychology as the research object, take the decomposition and integration of many music elements in the process of auditory cognition as the basic problem, and take the correspondence between roll and pitch name as the primary task.

\section{THE ORIENTATION OF PAYING ATTENTION TO AESTHETIC PSYCHOLOGY}

\subsection{Highlight the core purpose}

With the continuous improvement of the development of solfeggio discipline, the connotation and extension of the curriculum also constantly obtain new content and significance under the guidance of the new ideas of teaching reform. The understanding of solfeggio discipline should get rid of the old view of "spectrum recognition class", but make the discipline become a bridge connecting various disciplines and practices of music, and give play to the true significance of music auditory training. For one thing, solfeggio teaching should focus on the basic problem of the decomposition and integration of music elements in the process of auditory cognition; for another, it should overcome the limitations of physical auditory cognition of music elements such as high, low, long, short, strong and weak. Therefore, it can let music elements return to cultural attributes, promote their integration in auditory aesthetics, and realize the goal of music elements "from works to works".

Due to synaesthesia, the aesthetic correspondence between auditory experience and non-auditory experience will be generated unconsciously. There is no pure auditory experience in the form of sound, and auditory aesthetic synaesthesia will form some correspondence between the characteristics of constructing music elements and the modality. Mr Zhou Haihong pointed out in his monograph Music and the World that it Represents that there are six kinds of perceptual correspondence laws related to music hearing, including the synaesthesia related to pitch, intensity, time, time rate, tensity and heterosexual experience [5]. These six kinds of corresponding relations are all closely combined with the performance characteristics of various musical elements. Synesthesia is the medium through which auditory experience and emotional experience can be transformed, as sound forms without visual and semantic meanings can represent visual and conceptual objects. In the implementation of solfeggio course, auditory techniques are often mentioned, while auditory experience brought by "synaesthesia" psychology is ignored. However, the correspondence between acoustic forms and human modal activities is based on the characteristics of pitch, intensity, tone color and other elements, as well as the charm of musical culture displayed by these characteristics. These should be the basic content that the music education system should convey to students, and also an unavoidable task of solfeggio course as the basic music science teaching.

\subsection{Embody the aesthetic appeal}

Pitch hearing can bring people different degrees of hearing experience physiologically. The treble makes people excited, happy, positive and upward, describing agile and dextrous object movement, while the bass makes people depressed, sad, gloomy and deep, describing dull and clumsy. The rhythm stems from nature. The perception of the musical rhythm is a physiological reaction first, and then a corresponding concept will be formed, that is, fast rhythm will make people excited, and on the contrary, soothing. The speed will bring people different sense of space in auditory and psychological level. The long, slow and comfortable speed will bring people a sense of open space and calm and quiet emotions. The short, fast and dense speed will bring people a sense of narrow space as well as impatient and excited. The strength of the intensity will also bring people a corresponding auditory experience: fortissimo makes people feel heavy and near, while weak tone is light and far away. Tone is one of the main 
characteristics of sound. Due to the different sound elements, the difference in the quantity, quality and intensity of the overtones will produce different characteristics of sound. Pure timbre is considered friendly and mixed timbre is considered warm. Different timbre of musical instruments will show different aesthetic appeals under different cultural backgrounds.

Music elements can not be isolated from the joint perceptual psychology, and they always show a highly generalized beauty after being highly integrated by auditory thinking. As a member of the music education system of senior teachers, solfeggio teaching should follow the basic rules of music education, and simultaneously realize the auditory aesthetic in the analysis and integration of music elements. The development of solfeggio course should break the single pursuit of auditory technology, give the aesthetic demands in the process of auditory cognition, and reflect the cultural attributes of the solfeggio discipline.

\section{THE ORIENTATION OF FOCUSING ON AUDITORY THINKING}

\subsection{Realize the function of curriculum}

As a kind of emotion art, the extension of its sound form aims to perfect the emotional experience of the auditory subject in time. The characteristic display of these musical elements, such as the ups and downs of pitch, the relaxation of rhythm, the strength and the intensity of color, paints a beautiful picture for the listener and causes endless reveries

Based on this characteristic of music art, it is not difficult to see that the teaching of solfeggio for music elements cannot divest the auditory main body's appeal for "aesthetic feeling". The performance of different degrees of "beauty" is closely related to the cultural background associated with the listener and sound form. In the teaching of solfeggio, the author attaches importance to the role of music elements and actively explores the characteristics and values of music elements, that is, through the auditory training of music elements, the author obtains the ability to recognize and use them, as well as the ability to grasp and deal with many problems related to music aesthetics. This is not only directly related to the orientation of solfeggio course, but also related to the idea, content, purpose and method of music education in normal colleges to the transmission of basic music education.

Although musical elements are only the "bricks and tiles" of musical works, their value can not be underestimated, since it is the starting point of auditory aesthetics. As mentioned above, the decomposition and integration of music elements is the core content of the development of music auditory ability. The recognition and application of music elements are not only reflected in the level of auditory technology, but also in the whole process of auditory aesthetic. The two jointly constitute the main content of auditory thinking.

The auditory cognition of music elements cannot leave the important psychological matrix of "thinking". The operation process of auditory thinking is the integration of memory and imagination activities. The music elements are characterized in memory in the form of symbols, and are extracted and utilized at any time. These remembered music elements then form conceivable materials in the brain, and decompose and integrate the music information in memory.

Auditory thinking resides in every moment of music activity. It can be said that the process of acoustics cognition is the process of auditory thinking. It can be the thinking for external acoustics or the thinking for inner auditory sense. Curriculum development, as the "beacon" to regulate and guide curriculum objectives, curriculum contents and curriculum implementation, should start with the characteristics of "teacher education" and fully reflect the framework and macroscopic guiding significance of the subject. Solfeggio course is not only a "music reading course", but a "intensive training course" about music auditory thinking. The goal of the solfeggio course should establish the concept of "taking auditory thinking as the core of training", and build the thinking ability, thinking habit and thinking quality for the auditory subject from the accumulation of musical elements such as pitch and rhythm bit by bit.

\subsection{Update course philosophy}

The basic nature of the solfeggio course is not reflected in the training program on the music elements involved in the course content, but in the quality that can provide good auditory thinking for the music practice. The learning purpose of the solfeggio course is not limited to the listening and singing of music vocabulary, but to guide auditory individuals to master methods, develop good auditory habits, and provide a blueprint for auditory thinking for their future teaching activities.

From the general law of education, students' learning and development is a dynamic generation transformation process. The training content in view of music elements is just a carrier, which helps hearing individuals acquire certain hearing technology and aesthetic ability, turn this ability into the future teaching, and pass it on to students, which is the development direction of solfeggio course in normal universities. Aural thinking is the carrier of music thinking activities. Music thinking can be the harmonic thinking, polyphonic thinking, structure thinking, tone and color thinking related to the sound form ontology, but also the cognition and understanding related to the emotional experience and style grasp.

Music thinking starts from the formal logic thinking of acoustics and extends to the thinking of musical artistic expression. Therefore, only on the basis of the sense, perception and memory of acoustics, can students carry out comprehensive musical thinking under the guidance of rationality. Acoustics and its structure are the direct object 
of thinking, and it is the premise and foundation of the whole music thinking [6]. Acoustics and its structure are the direct object of thinking, and it is the premise and foundation of the whole music thinking. This kind of music is full of artistry, since all the details of musical behavior are induced by the normative logic. Therefore, such music must be able to reflect the accumulation of the entire music history, showing the style of The Times and the national interest [7].

In the actual teaching, it is necessary to strengthen the training of the form logic thinking of sound form consciously and purposefully, and take the perception of pitch, the memory of melody, the imitation of rhythm, the identification of timbre, the mastery of speed, the control of strength and the experience of the beauty of music form and the beauty of modality as the main training content. The assessment data and experience prove that it is beneficial to promote the auditory subject to conduct "independent" musical behavior, and to realize their own aesthetic appeal at the level of rational expression. If teachers can penetrate the details of these daily contents of solfeggio teaching, implement the corresponding ideas and re-examine the definition, category, purpose and function of the course itself, new guidance and ideas will be generated for the development of solfeggio teaching.

\section{CONCLUSION}

In summary, The author believes that in exploring the development of solfeggio course in normal universities, first, it is necessary to focus on the essence of the subject; second, it is necessary to pay attention to aesthetic psychology and break through the old curriculum concept.
It is worth emphasizing that the identification of music elements is not the ultimate goal. The ability to express the beauty of music, understand the meaning of music, and transfer the auditory culture of music is the responsibility of auditory thinking and the basis of promoting the development of solfeggio courses.

\section{REFERENCES}

[1] Li Songlin. Four Practice Points of Deep Teaching

[J]. Educational Theory and Practice, 2014 (31): 54.

[2] Bennett Reimer, Xiong Lei. Philosophy of Music Education [M]. Beijing: People's Music Press, 2003.

[3] Б·M· Jeplov, Sun Ye. Psychology of Music Ability [M]. Beijing: People's Education Press, 1990.

[4] Lei Guangyao. Thoughts on the Solmization of Solfeggio and its Selection [J]. Chinese Music, 2013 (1): 184-185.

[5] Zhou Haihong. The World of Music and its Performance [M]. Beijing: Central Conservatory of Music Press, 2004: 50.

[6] Li Xiaonuo. Cognitive and Psychology of Music [M]. Guilin: Guangxi Normal University Press, 2017: 228.

[7] Lin Hua. How Musicians "Think" [M]. Shanghai: Shanghai Music Press, 2018: 31. 